\title{
Journal of Computational Mathematica
}

\section{Open Access}

\section{Skolem Mean Labeling of Four Star Graphs \\ $K_{1, a_{1}} \cup K_{1, a_{2}} \cup K_{1, a_{3}} \cup K_{1, b}$ where $\left|b-\left(a_{1}+a_{2}+a_{3}\right)\right|=4$}

Ramesh DST ${ }^{1 *}$ and Sopna $\mathrm{SO}^{2}$

${ }^{1}$ Department of Mathematics, Margchosis College, Nazareth, Manonmaniam Sundaranar University, Tirunelveli - 627012, Tamil Nadu, India.

${ }^{2}$ Department of Mathematics, The American College, Madurai, Manonmaniam Sundaranar University, Tirunelveli - 627012, Tamil Nadu, India.

\begin{abstract}
In this paper, we prove that four star graph $G=K_{1, a_{1}} \cup K_{1, a_{2}} \cup K_{1, a_{3}} \cup K_{1, b}$ where $a_{1} \leq a_{2} \leq a_{3}$ is a skolem mean graph if $\left|b-\left(a_{1}+a_{2}+a_{3}\right)\right|=4$.
\end{abstract}

Key words: Skolem mean graph, skolem mean labeling, star graphs.

AMS classification: 39A10, 39A11, 39A13, 39A70, 49M.

\section{Introduction}

In this paper all graphs are finite, simple and undirected. Terms and notations are used in the sense of Harary [3]. Much work is done by many researchers on skolem mean labelling [1], [2] and [3]. In [5], [6] and [7] some results are proved in four star graph $G=K_{1, a_{1}} \cup K_{1, a_{2}} \cup K_{1, a_{3}} \cup K_{1, b}$ on skolem mean labelling. In this paper, we prove that four star graph $G=K_{1, a_{1}} \cup K_{1, a_{2}} \cup K_{1, a_{3}} \cup K_{1, b}$ where $a_{1} \leq a_{2} \leq a_{3}$ is a skolem mean graph if $\left|b-\left(a_{1}+a_{2}+a_{3}\right)\right|=4$. That is when $b=\left(a_{1}+a_{2}+a_{3}\right)+4$ and $b=\left(a_{1}+a_{2}+a_{3}\right)-4$.

Definition 1.1 A graph $G=(V, E)$ with $p$ vertices and q edges is said to be a skolem mean graph if there exists a function $f$ from the vertex set of $G$ to $1,2, \cdots, p$ such that the induced map $f^{*}$ from the edge set of $G$ to $2,3, \cdots, p$ defined by

$f^{*}(e=u v)= \begin{cases}\frac{f(u)+f(v)}{2} & \text { if } f(u)+f(v) \text { is even } \\ \frac{f(u)+f(v)+1}{2} & \text { if } f(u)+f(v) \text { is odd }\end{cases}$

the resulting edges get unique labels from the set $2,3, \cdots, p$.

Theorem 1.2 The four star $G=K_{1, a_{1}} \cup K_{1, a_{2}} \cup K_{1, a_{3}} \cup K_{1, b}$ where $a_{1} \leq a_{2} \leq a_{3}$

1*dstramesh@gmail.com, ${ }^{2}$ s.sopna@yahoo.com

Page 75 of 79 
is a skolem mean graph if $\left|b-\left(a_{1}+a_{2}+a_{3}\right)\right|=4$. Proof: Let $A_{i}=\sum_{k=1}^{i} a_{k}$. That is,
$A_{1}=a_{1} ; A_{2}=a_{1}+a_{2}$ and $A_{3}=a_{1}+a_{2}+a_{3}$. Consider the graph $G=K_{1, a_{1}} \cup K_{1, a_{2}} \cup K_{1, a_{3}} \cup K_{1, b}$. Let $V=\bigcup_{k=1}^{4} V_{k}$ be the vertex set of $\mathrm{G}$ where $V_{k}=\left\{v_{k, i}: 0 \leq i \leq a_{k}\right\}$ for $1 \leq k \leq 3$ and $V_{4}=\left\{v_{4, i}: 0 \leq i \leq b\right\}$. Let $E=\bigcup_{k=1}^{4} E_{k}$ be the edge set of $\mathrm{G}$ where $E_{k}=\left\{v_{k, 0} v_{k, i}: 0 \leq i \leq a_{k}\right\}$ for $1 \leq k \leq 3$ and $E_{4}=\left\{v_{4,0} v_{4, i}: 0 \leq i \leq b\right\}$.

The condition $\left|b-\left(a_{1}+a_{2}+a_{3}\right)\right|=4 \Rightarrow b=A_{3}-4$ or $b=A_{3}+4$.

That is, there are two cases viz. $b=A_{3}-4$ and $b=A_{3}+4$.

Let us prove in each of the two cases the graph $G$ is a skolem mean graph.

Case 1: Let $b=A_{3}+4$

$\mathrm{G}$ has $A_{3}+b+4=2 A_{3}+8$ vertices and $A_{4}+b=2 A_{3}+4$ edges.

The vertex labeling $f: V \rightarrow\left\{1,2, \cdots, A_{3}+b+4=2 A_{3}+8\right\}$ is defined as follows:

$$
\begin{array}{rlrl}
f\left(v_{1,0}\right) & =1 ; \quad f\left(v_{2,0}\right)=2 ; & f\left(v_{3,0}\right)=4 ; \\
f\left(v_{4,0}\right) & =A_{3}+b+3=2 A_{3}+7 \\
f\left(v_{1, i}\right) & =2 i+4 & 1 \leq i \leq a_{1} \\
f\left(v_{2, i}\right) & =2 A_{1}+2 i+4 & 1 \leq i \leq a_{2} \\
f\left(v_{3, i}\right) & =2 A_{2}+2 i+4 & 1 \leq i \leq a_{3} \\
f\left(v_{4, i}\right) & =2 i+11 \leq i \leq b-2=A_{3}+2 \\
f\left(v_{4, b-1}\right) & =A_{3}+b+2=2 A_{3}+6 \\
f\left(v_{4, b}\right) & =A_{3}+b+4=2 A_{3}+8
\end{array}
$$

The corresponding edge labels are as follows:

The edge label of $v_{1,0} v_{1, i}$ is $3+i$ for $1 \leq i \leq a_{1}$ (edge labels are $\left.4,5, \cdots, a_{1}+3=A_{1}+3\right), v_{2,0} v_{2, i}$ is $A_{1}+3+i$ for $1 \leq i \leq a_{2}$ (edge labels are $\left.A_{1}+4, A_{1}+5, \cdots, A_{2}+3\right), v_{3,0} v_{3, i}$ is $A_{2}+4+i$ for $1 \leq i \leq a_{3}$ (edge labels are $\left.A_{2}+5, A_{2}+6, \cdots, A_{3}+4\right), v_{4,0} v_{4, i}$ is $A_{3}+4+i$ for $1 \leq i \leq b-2=A_{3}+2$ (edge labels are $\left.A_{3}+5, A_{3}+6, \cdots, 2 A_{3}+6\right), v_{4,0} v_{b-1}$ is $2 A_{3}+7$ and $v_{4,0} v_{4, b}$ is $2 A_{3}+8$.

These induced edge labels of graph $G$ are unique. 
ISSN: 2456-8686, Volume 2, Issue 1, 2018:75-79

DOI : http://doi.org/10.26524/cm29

Hence $G$ is a skolem mean graph.
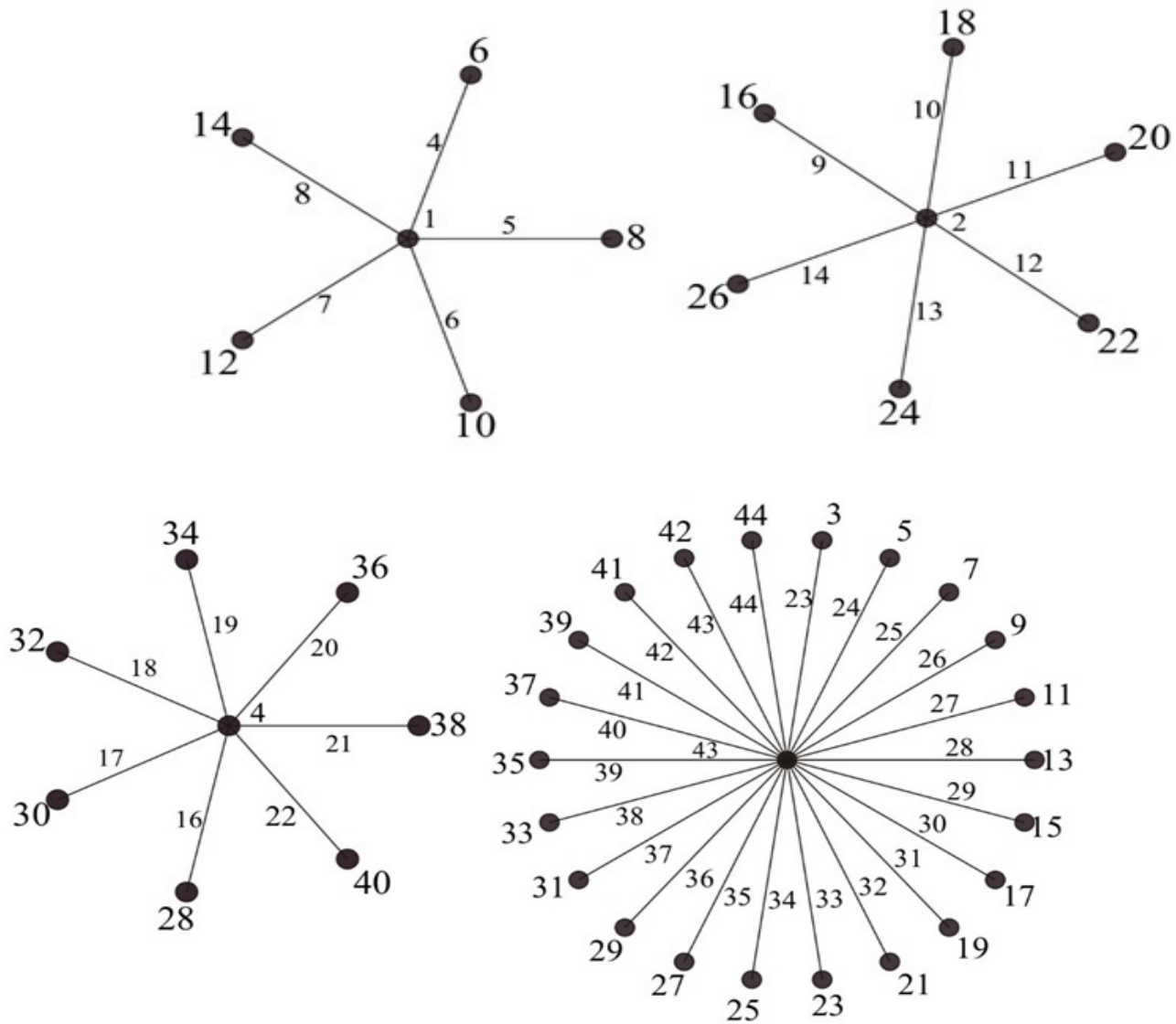

Figure 1: $K_{1,5} \cup K_{1,6} \cup K_{1,7} \cup K_{1,22}$

Case 2: Let $b=A_{3}-4$

$G$ has $A_{3}+b+4=2 A_{3}$ vertices and $A_{3}+b=2 A_{3}-4$ edges.

The vertex labeling $f: V \rightarrow\left\{1,2, \cdots, A_{3}+b+4=2 A_{3}\right\}$ is defined as follows:

$$
\begin{array}{llrl}
f\left(v_{1,0}\right) & =2 ; \quad f\left(v_{2,0}\right)=4 ; & f\left(v_{3,0}\right)=6 ; \\
f\left(v_{4,0}\right) & =A_{3}+b+4=2 A_{3} & \\
f\left(v_{1, i}\right) & =2 i-1 & & 1 \leq i \leq a_{1} \\
f\left(v_{2, i}\right) & =2 A_{1}+2 i-1 & & 1 \leq i \leq a_{2} \\
f\left(v_{3, i}\right) & =2 A_{2}+2 i-1 & & 1 \leq i \leq a_{3} \\
f\left(v_{4, i}\right) & =2 i+6 & & 1 \leq i \leq b
\end{array}
$$

The corresponding edge labels are as follows:

The edge label of $v_{1,0} v_{1, i}$ is $1+i$ for $1 \leq i \leq a_{1}$ (edge labels are $\left.2,3, \cdots, a_{1}+1=A_{1}+1\right), v_{2,0} v_{2, i}$ is $A_{1}+2+i$ for $1 \leq i \leq a_{2}$ (edge labels are 
$\left.A_{1}+3, A_{1}+4, \cdots, A_{2}+2\right), v_{3,0} v_{3, i}$ is $A_{2}+3+i$ for $1 \leq i \leq a_{3}$ (edge labels are $\left.A_{2}+4, A_{2}+5, \ldots, A_{2}+a_{3}+3=A_{3}+3\right), v_{4,0} v_{4, i}$ is $A_{3}+3+i$ for $1 \leq i \leq b=A_{3}-4$ (edge labels are $A_{3}+4, A_{3}+5, \cdots, A_{3}+3+b=A_{3}+3+A_{3}-4=2 A_{3}-1$ ).

These induced edge labels of graph $\mathrm{G}$ are unique.

Hence $G$ is a skolem mean graph.

\section{Example 1.3}
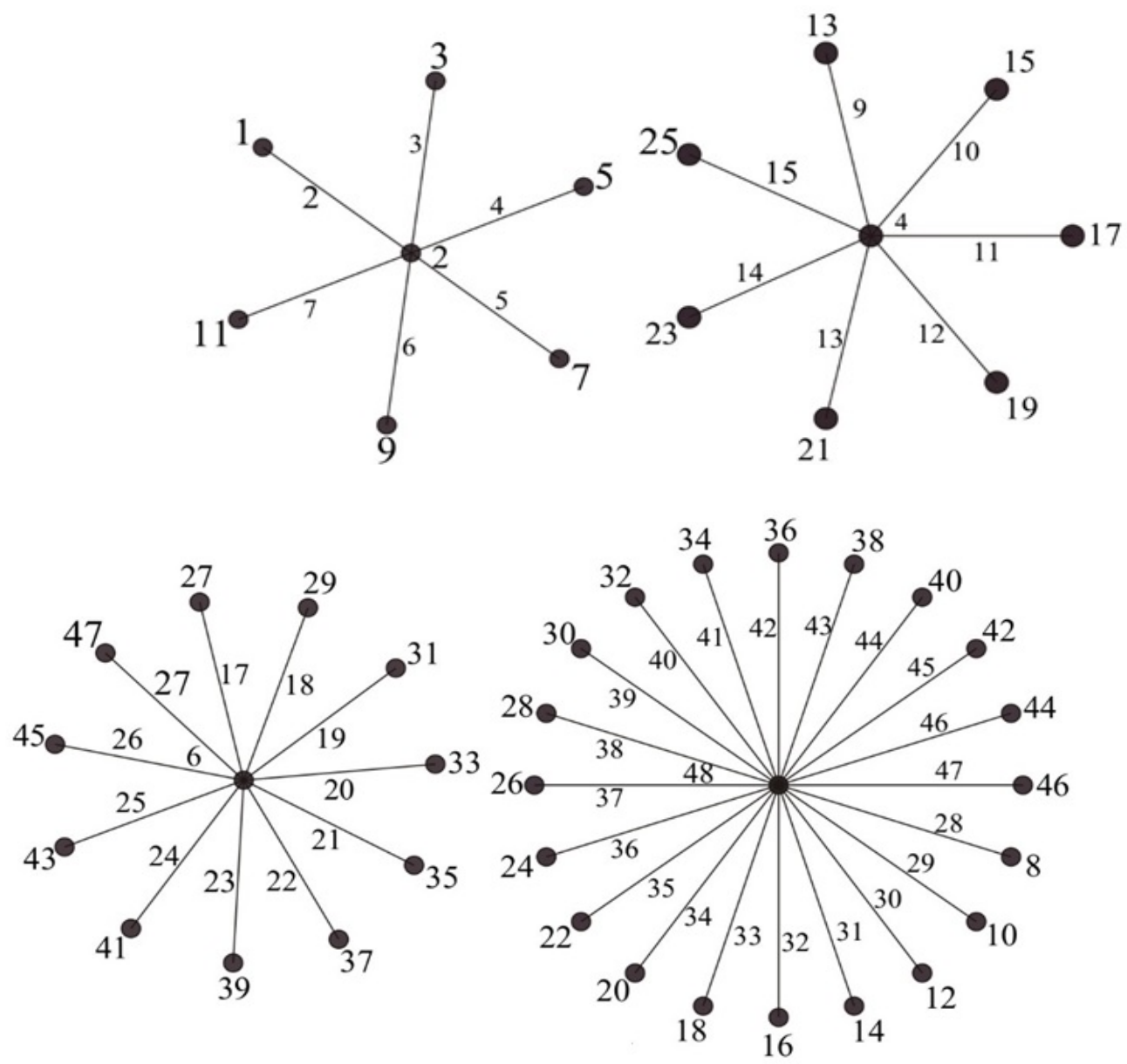

Figure 2: $K_{1,6} \cup K_{1,7} \cup K_{1,1} \cup K_{1,20}$

\section{References}

[1] Balaji V, Ramesh DST and Subramanian A, Skolem Mean Labeling, Bulletin of Pure and Applied Sciences, 26(2), 2007, 245-248.

[2] Balaji V, Ramesh DST and Subramanian A, Some Results on Skolem Mean graphs, Bulletin of Pure and Applied Sciences, 27(1), 2008, 67-74. 
ISSN: 2456-8686, Volume 2, Issue 1, 2018:75-79

DOI : http://doi.org/10.26524/cm29

[3] Gallian JA, A Dynamic Survey of Graph Labeling, The Electronic Journal of Combinatorics, 14, 2016, \#DS6.

[4] Harary F, Graph Theory, Addison- Wesley, Reading Mars, (1972).

[5] Ramesh DST, Sopna SO, Gnanaselvi I and Syed Ali MP, Nisaya Skolem Mean Labeling Of Four Star Graphs $K_{1, a_{1}} \cup K_{1, a_{2}} \cup K_{1, a_{3}} \cup K_{1, b}$ where $a_{1}+a_{2}+a_{3}+2 \leq b \leq a_{1}+a_{2}+a_{3}+3$, International Journal of Scientific Research, 6(8), 2017, 190-193.

[6] Ramesh DST, Sopna SO and Gnanaselvi I, Skolem Mean Labeling Of Four Star Graphs $K_{1, a_{1}} \cup K_{1, a_{2}} \cup K_{1, a_{3}} \cup K_{1, b}$ where $a_{1}+a_{2}+a_{3}-1 \leq b \leq a_{1}+a_{2}+a_{3}+1$, IOSR Journal of Engineering (IOSRJEN), 7(7), July 2017, 05-11.

[7] Ramesh DST, Sopna SO and Gnanaselvi I, Skolem Mean Labeling Of Four Star Graphs $K_{1, a_{1}} \cup K_{1, a_{2}} \cup K_{1, a_{3}} \cup K_{1, b}$ where $a_{1}+a_{2}+a_{3}-3 \leq b \leq a_{1}+a_{2}+a_{3}-2$, International Journal of Engineering Research and Applications (IJERA), 7(9), 2017, 29-32. 